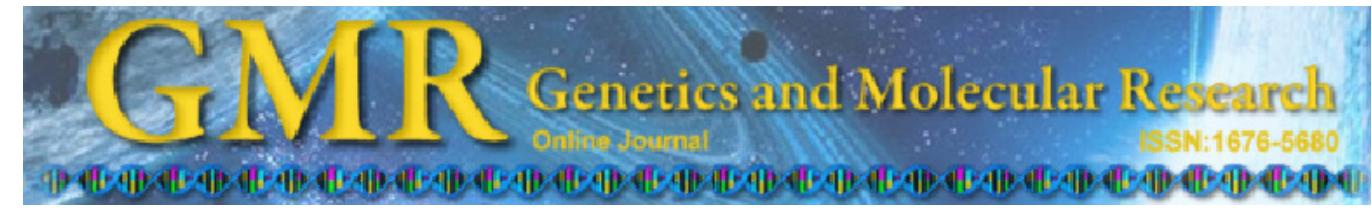

Case Report

\title{
Familial balanced translocation leading to an offspring with phenotypic manifestations of 9p syndrome
}

\author{
L.S. Abreu ${ }^{1}$, M.S. Brassesco ${ }^{2}$, M.L.C. Moreira ${ }^{3}$, J.M. Pina-Neto ${ }^{1,3}$ \\ ${ }^{1}$ Departamento de Genética, Faculdade de Medicina de Ribeirão Preto, \\ Universidade de São Paulo, Ribeirão Preto, SP, Brasil \\ ${ }^{2}$ Departamento de Biologia, Faculdade de Filosofia, \\ Ciências e Letras de Ribeirão Preto, Ribeirão Preto, SP, Brasil \\ ${ }^{3}$ Divisão de Genética Médica, Hospital de Clínicas, \\ Universidade de São Paulo, Ribeirão Preto, SP, Brasil \\ Corresponding author: L.S. de Abreu \\ E-mail: ludiserafimabreu@usp.br
}

Genet. Mol. Res. 13 (2): 4302-4310 (2014)

Received July 24, 2013

Accepted December 6, 2013

Published June 9, 2014

DOI http://dx.doi.org/10.4238/2014.June.9.16

\begin{abstract}
We report two similarly affected cousins (children of monozygotic twin sisters) with phenotypic features consistent with $9 \mathrm{p}$ deletion syndrome, including dysmorphic craniofacial features (trigonocephaly, midface hypoplasia, upward-slanting palpebral fissures and long philtrum), intellectual disability and disorders of sex development. Initial cytogenetic examination showed normal karyotypes in the probands and their respective parents, though multiplex ligation probe amplification revealed a 1q terminal duplication and a $9 p$ terminal deletion in both affected children. Further analysis by fluorescence in situ hybridization, identified a familial balanced cryptic translocation $\mathrm{t}(1 ; 9)(\mathrm{q} 44 ; \mathrm{p} 23)$ in the mothers, showing the importance of the association of molecular cytogenetic techniques in clinical genetics, given the implications for the care of
\end{abstract}


patients and for genetic counseling.

Key words: Multiplex ligation proble amplification subtelomeric; Trigonocephaly; 9p deletion; Fluorescence in situ hibridization; Familial rearrangement; Genetic counseling

\section{INTRODUCTION}

Monosomy $9 \mathrm{p}$ syndrome is a chromosomal syndrome with cardinal features including psychomotor retardation, trigonocephaly, flat nasal bridge, long philtrum, short, broad and webbed neck, genital anomalies, and sex reversal (Alfi et al., 1973; Hoo et al., 1982; Swinkels et al., 2008). Other features frequently seen in this condition include hypertelorism, epicanthus, small palpebral fissures, anteverted nares, low-set malformed ears, micrognathia and hypotonia (Huret et al., 1988; Taylor et al., 1991; Shashi et al., 1998). Previous studies have delineated the size of 9p deletions in an attempt to develop genotype/phenotype correlations. After analysis of 24 patients with microscopically visible 9p deletion, Christ et al. (1999) suggested that the critical region in 9p-deletion syndrome could be assigned to a 4-6-Mb region in 9p22- p23. More recently, Swinkels et al. (2008) distinguished two different groups among patients; one encompassing patients with the clinically recognizable 9p deletion phenotype (consensus phenotype consisting of trigonocephaly, small palpebral fissures, flat nasal bridge with anteverted nostrils, long philtrum, and micro/retrognathia), and a second group encompassing those patients who, despite being positive for the deletion, did not meet the criteria for the consensus phenotype, particularly lacking trigonocephaly. The critical region for the consensus phenotype was subsequently mapped to the 9p22.3 region (Swinkels et al., 2008). Genital and/or gonadal disorders are also observed in male individuals who present with $9 p$ deletion, though occurring at a more distal 9p24.3 region, extending from the DMRT genes to the telomere (Barbaro et al., 2008). In XY patients, sex development disorders display a wide spectrum of presentation, in which the external genitalia can range from complete female to male phenotype with hypospadias, while the gonadal phenotype can vary from complete gonadal dysgenesis to ovotestes and to cryptorchid and/or hypoplastic testis (Barbaro et al., 2009).

$D M R T$ genes (DMRT1, DMRT2, and DMRT3) have been suggested as the strongest candidates for the gonadal dysgenesis phenotype (Calvari et al., 2000; Kawara et al., 2006), but the molecular mechanism underlying gonadal dysgenesis is still not clear (Swinkels et al., 2008). The mechanism could involve haploinsufficiency of one or more genes in the deleted region or unmasking of a recessive mutation on the other chromosome, or it could be more complicated (Barbaro et al., 2009). In this case report, we describe a cryptic chromosomal rearrangement in two cousins similarly affected with partial $9 \mathrm{p}$ monosomy and partial 1q trisomy, both originating from a balanced translocation $\mathrm{t}(1 ; 9)(\mathrm{q} 44 ; \mathrm{p} 24.3)$ present in both mothers. Although monosomy $9 \mathrm{p}$ has been seldom reported (Swinkels et al., 2008), some cases of 1q44 duplication have been described (Lenzini et al., 2009), while reported cases with both alterations, partial monosomy 9p and partial trisomy 1q are even rarer (Verbraak et al., 1992; Kulikowski et al., 2008).

\section{MATERIAL AND METHODS}

\section{Selection of subjects}

The patients were seen at the Human Genetics Ambulatory Service of the Hospital das 
Clínicas, Faculdade de Medicina de Ribeirão Preto, Universidade de São Paulo. This study was carried out under protocols approved by the Ethics Committee of the Universidade de São Paulo, according to HCRP No. 12460/2010. Clinical data of the patients were obtained either by physical examination, familial history or thorough review of medical history.

\section{Clinical characteristics of patients}

Patient 1 was born to a 20 -year-old mother, first pregnancy without history of abortion and a 25 -year-old father. The child was born at 39 weeks gestation by cesarean section. His birth weight was $3.4 \mathrm{~kg}$ with a birth length of $51.5 \mathrm{~cm}$. He showed stenosis of the pylorus corrected at 22 days, umbilical herniorrhaphy corrected at three months of life, craniosynostosis corrected at 5 years and trigonocephaly in addition to global hypotonia, hyporeflexia, mild cerebral atrophy, and a clinical picture of developmental delay. Currently, he is 8 years and 10 months old and has idiopathic intellectual disability, muscular atrophy and dysmorphic features including high skull, brachycephaly, frontal plane, bitemporal narrowing, arched eyebrows, low nasal root, anteverted nares, long philtrum, small ears with unfolded helix, small mouth, high palate and narrow, irregularly positioned teeth, pectus carinatum, long and fine fingers without arachnodactyly, discrete camptodactyly of the 3rd and 5th fingers, long toes with rounded extremities, increased interval between the 1st and 2nd toes, and left cryptorchidism. Six years later, the monozygotic twin sister at age 26 gave birth to the second patient (Patient 2) with similar features as his cousin. It was also the first pregnancy without history of abortion. The father was 28 years old. The patient was born at 40 weeks gestation by cesarean section. His birth weight was $3.9 \mathrm{~kg}$ with a birth length of $51.5 \mathrm{~cm}$. First, the presence of hypotonia, trigonocephaly and cardiopathy (CIA + PCA) was observed. The craniofacial dysmorphisms noted were high skull, corrected front wide craniosynostosis, low-set hair on forehead, arched eyebrows, small eyes with apparent blepharophimosis, short eyelashes, ears low-set and later small and round, low nasal root, anteverted nostrils, long philtrum, ogival palate and retrognathia.

Regarding the digits, the following features were identified: long and thin fingers without arachnodactyly, bilateral camptodactyly of the 5th finger, adducted thumbs, long toes with rounded ends, hallux valgus, pseudocamptodactyly, the 3rd toe overlapping the 4th, and clinodactyly of toes. In relation to the genitals, micropenis, bilateral cryptorchidism and hypoplastic scrotum were observed. Currently, Patient 2 is 1 year and 4 months old and shows developmental delay.

\section{Cytogenetic studies}

Metaphase chromosomes were prepared by a standard protocol: $0.5 \mathrm{~mL}$ peripheral blood was added to $10 \mathrm{~mL}$ RMPI 1640 medium (Sigma Chemical Co., St. Louis, MO, USA) supplemented with $20 \%$ fetal bovine serum, $2 \%$ PHA and penicillin/streptomycin (Gibco BRL, Gaithersburg, MD, USA). The cultures were incubated for $72 \mathrm{~h}$ at $37^{\circ} \mathrm{C}$ and treated with colchicine $(0.56 \%)$ for the last $30 \mathrm{~min}$. The cells were harvested and slides prepared according to a conventional method. The chromosomes were GTG-banded, and 20 metaphases with a minimum quality of 550 bands of resolution were examined (Seabright, 1971). 


\section{Multiplex ligation-probe amplification (MLPA) for screening of subtelomeric rearrangements}

A specifically designed set of probes for testing subtelomeric imbalances in the SALSA P036B and P070 Human Telomere test kits (MRC-Holland, Amsterdam, The Netherlands) was used. If the patient shows any alteration detected by MLPA test, the parents will be called to perform the same examination with the objective to exclude the possibility of the change to be non-pathogenic polymorphism.

The MLPA mix contained probes for all subtelomeric regions except the short arms of the acrocentric chromosomes (13p, 14p, 15p, 21p, and 22p), for which probe recognition sequences were on the q arm, in one of the genes just proximal to the telomeric repeats. Sequences detected by two probe mixes were different from each other. MLPA analysis was performed following manufacturer instructions. Amplification products were identified and quantified by capillary electrophoresis on an ABI 3130 Genetic Analyzer (Applied Biosystems, Foster City, CA, EUA). The fluorescent signal strength of the PCR products was determined by the GeneMapper software version 3.4 and the integrated peaks height were exported to a Coffalyser software version 8.0 for further calculations. For each patient, the normalized peak pattern of each subtelomeric region was divided by the average peak pattern of all samples $(\mathrm{N}>20)$ in the same experiment. The resulting values were approximately 1.0 for wild type peaks, $<0.70$ for deletions, and $>1.3$ for duplications.

\section{Fluorescence in situ hybridization}

Fluorescence in situ hybridization (FISH) technique was performed using probes for subtelomeric region 9pter (KBI-40217/Red) and for subtelomeric region 1qter (KBI-40202/ Green) according to the protocol provided by the manufacturer (Kreatech's Poseidon ${ }^{\mathrm{TM}}$, Amsterdam, The Netherlands). The preparations were dehydrated, dried, and counterstained with DAPI (Sigma Chemical Co.) plus antifade and analyzed with a fluorescence microscopy.

\section{RESULTS}

Metaphases of each individual were cytogenetically examined by GTG banding. Patients 1 and 2 and their respective parents showed a normal complement of chromosomes. The MLPA kits detected in both probands two cryptic changes corresponding to a deletion in the 9p24.3 region (probes 01727-L02050 and 02716-L00688 from the P036 and P070 kits, respectively) and a duplication in the 1q44 region (probes 02392-L02149 and 04084-L03605 from the P036 and P070 kits, respectively) (Figure 1), while the respective parents showed normal outcomes. Since both probands are first cousins and sons of two monozygotic twin sisters, it was suspected that the mothers would be carriers of a cryptic balanced translocation involving the $1 \mathrm{q}$ and $9 \mathrm{p}$ regions. To investigate this hypothesis, the FISH technique was applied using subtelomeric $9 \mathrm{p}$ and $1 \mathrm{q}$ probes in both probands and their mothers. In metaphase cells of the probands, only one signal of the $9 p$ probe was observed, showing hybridization on p24.3 of chromosome 9, while the 1q probe produced three signals, one located in the distal region of the short arm of chromosome der(9) and two signals in the q44 region of each chromosome 1 (Figure 2). 


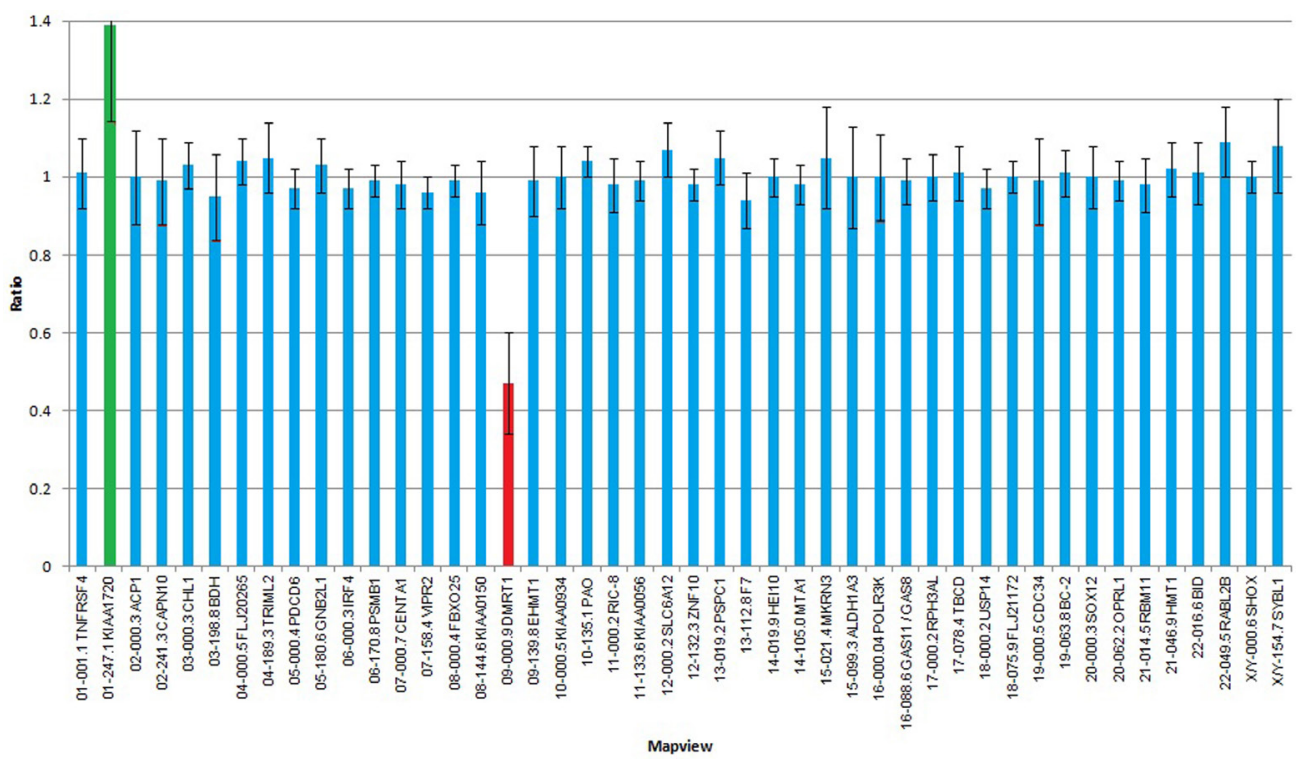

Figure 1. Duplication of the 1q44 region and deletion of the 9p24.3 region detected in both probands through P036 and P070 MLPA kits.

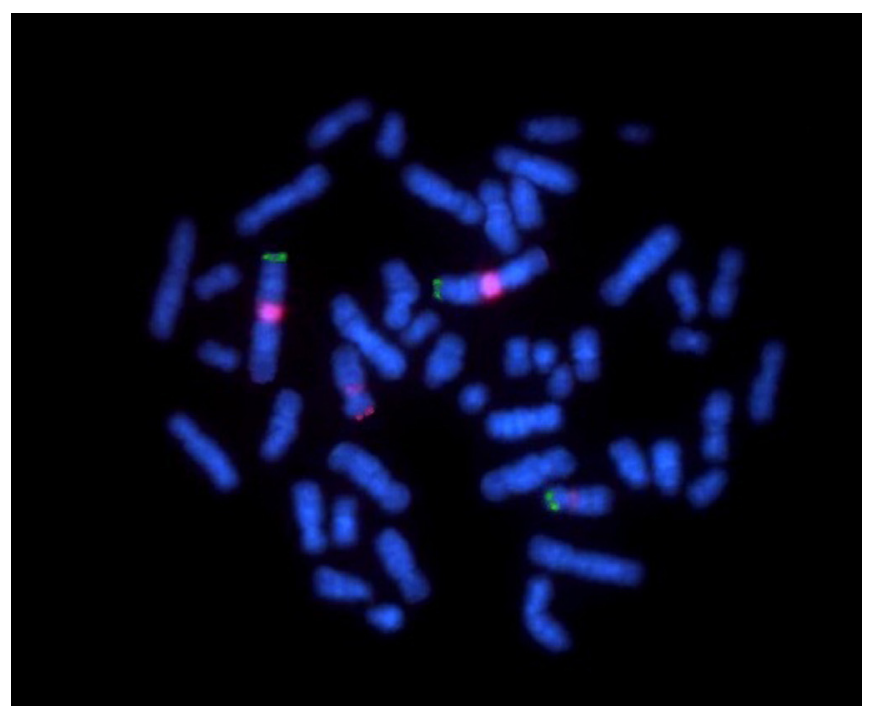

Figure 2. Metaphase cells of the probands: one signal of the probe $9 \mathrm{p} 24.3$ on the chromosome 9 , one signal of the probe 1q44 on the short arm of chromosome der(9), and two signals of the probe 1q44 on the chromosome 1.

In metaphase cells of both mothers, FISH signals of the 9p24.3 probe were identified on the normal chromosome 9 and on the long arm of der(1) and signals of the 1q44 probe on the normal chromosome 1 and on the short arm of der(9), revealing a balanced translocation (Figure $3 \mathrm{~A}$ and $\mathrm{B}$ ). 

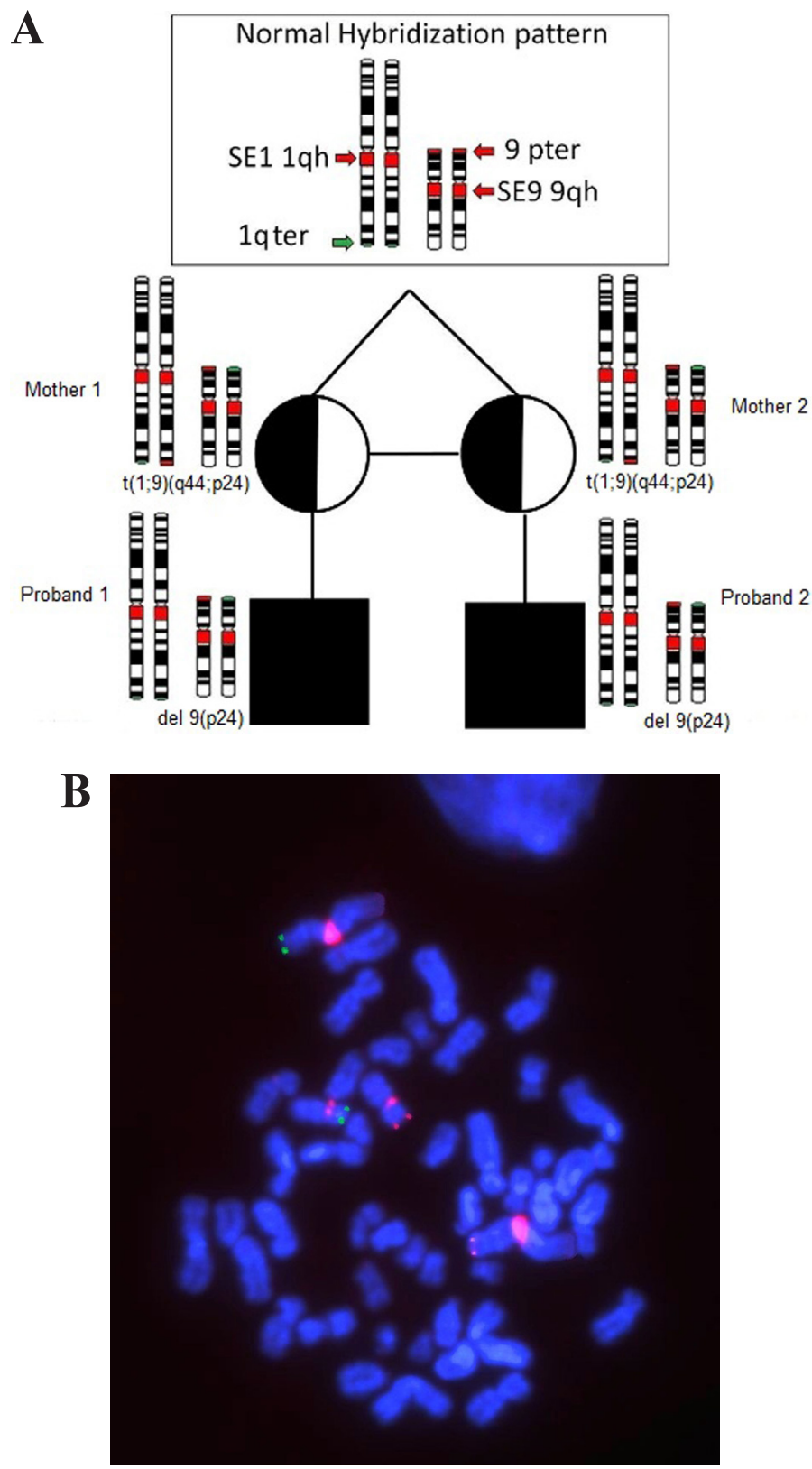

Figure 3. Two signals of the probe 9p24.3: one on the chromosome 9 and the other on the long arm of chromosome der(1). Two signals of the probe 1q44: one on the chromosome 1 and the other on the short arm of chromosome der(9), revealing a balanced translocation in both mothers (Figure $3 \mathrm{~A}$ and $\mathrm{B}$ ).

Therefore, it was possible to confirm in both probands a deletion of the short arm region of chromosome 9 and a trisomy of the distal portion of the long arm of chromosome 1, both cryptic changes, while a balanced submicroscopic translocation $\mathrm{t}(1,9)(\mathrm{q} 44 ; \mathrm{p} 24)$ was detected in the mothers. 


\section{DISCUSSION}

The MLPA technique with the P036 and P070 kits detected a 9pter monosomy and a 1qter trisomy in both probands. Despite the 9p24.3 deletion being microscopic and does not to include the critical region for the consensus phenotype of 9p syndrome (Swinkels et al., 2008), both cousins showed clinical features of this syndrome including neuropsychomotor delay, hypotonia, trigonocephaly, midface hypoplasia, upslanted palpebral fissures, flat nasal bridge, long philtrum, and small and malformed ears, in agreement with previous descriptions for this syndrome (Alfi et al., 1973; Huret et al., 1988; Calvari et al., 2000; Faas et al., 2007; Barbaro et al., 2009), which is in agreement with Hauge et al. (2008), who described that the minimal deleted region shared by their patients with clinically relevant phenotypes includes the first $2 \mathrm{Mb}$ of 9pter, region distal to the previously described critical region 9p22.3. Therefore, it is likely that phenotypic features attributed to the $9 \mathrm{p}$-deletion syndrome may be caused by multiple regions on 9p or other modifying factors in the genome (Hauge et al., 2008). Similarly, Barbaro et al. (2009) suggested that the mild cranial dysmorphism in patients with deletions distal to Swinkels' critical region could be caused by either misregulation of the candidate gene for trigonocephaly or the deletion of another gene(s) involved in craniofacial development. Thus, the findings in our patients support the observations made by Barbaro et al. (2009), suggesting a more distal critical region for the cranial dysmorphisms observed in 9p deletion syndrome. The use of two MLPA kits allowed the detection of the cryptic alteration and provided a better understanding of the size of the altered regions. Thus, it can be stated that the deletion in the region of the short arm of chromosome 9p has a minimum length of $\sim 1 \mathrm{Mb}$, where the genes $D O C K 8, K A N K 1$, and DMRT1 are located. Specifically, deletion of the DOCK8 gene has been associated with mental retardation (Chen et al., 2011). Griggs et al. (2008) also described two unrelated patients (one male and one female) with mental deficiency and developmental disability, who had a heterozygous disruption of the DOCK8 gene, in which the male had a genomic deletion of approximately $230 \mathrm{~kb}$ in subtelomeric $9 \mathrm{p}$, while the female carried a de novo balanced translocation $\mathrm{t}(\mathrm{X} ; 9)(\mathrm{q} 13.1 ; \mathrm{p} 24)$. Although those patients had a heterozygous disruption in the DOCK8 gene and not a deletion, the authors showed that this gene is associated with mental deficiency and developmental delay (Griggs et al., 2008). Alternatively, $K A N K 1$ is a maternally imprinted gene that is expressed only from the paternal allele (Lerer et al., 2005). Deletion of the KANK1 gene causes parent-of-origin-dependent inheritance of familial cerebral palsy that occurs only in individuals inheriting the deletion from the father (Lerer et al., 2005). Alterations involving the candidate sex-determining gene DMRT1 are often associated with sex reversal and gonadal dysgenesis (Smith et al., 1999; Shan et al., 2000). Defective testicular development and XY feminization occur when this gene is present in hemizygosis (Shan et al., 2000). Moreover, a great variability in impaired development in male cases of monosomy $9 \mathrm{p}$ has been reported, confirming the cryptorchidism and micropenis observed in Patient 1 and Patient 2, respectively.

Conversely, $9 \mathrm{p}$ deletions may also originate from rearrangements involving other chromosomes. Huret et al. (1988) reported 11 new cases of 9p deletion and reviewed 69 previously published cases. Of those 80,39 had a "pure" 9p deletion and 41 were associated with another, unbalanced chromosome segment, as in our patients, resulting from a balanced translocation between chromosomes 1 and 9 in their mothers. However, partial 1q trisomy syndrome is a rare chromosomal abnormality, and because of the differences in the extension 
of the duplicated 1q segment and the frequent concomitant presence of other chromosome imbalances, the patient phenotypes are quite variable, making it difficult to delineate a syndrome phenotype (Kulikowski et al., 2008). Bartsch et al. (2001) performed a comparative delineation of the $1 \mathrm{q}$ duplication phenotype and reported that large $(1 \mathrm{q} 21 \rightarrow \mathrm{qter})$ as well as proximal $(1 \mathrm{q} 11 / 12 \rightarrow \mathrm{q} 22-25)$ and interstitial $(1 \mathrm{q} 25 \rightarrow \mathrm{q} 31-41)$ duplications coincided with more severe visceral malformations, reduced life expectancy and more severe mental retardation. On the other hand, terminal duplications $(1 \mathrm{q} 32 \rightarrow \mathrm{qter})$ are associated with less severe visceral malformations, longer survival, but also severe mental retardation, while small terminal duplications $(1 \mathrm{q} 42 \rightarrow$ qter) show mild dysmorphisms, with intellectual performance being mostly within the normal range (Bartsch et al., 2001). Our results obtained through the use of P036 and P070 MLPA kits allow us to assume that the region involved in 1q rearrangement has a minimum size of $\sim 2.20 \mathrm{Mb}$, which cannot be identified by cytogenetic examination with resolution (500 bands) that in theory can only detect changes $\sim 5.0 \mathrm{Mb}$ in size. Rooms et al. (2006) described a girl without mental retardation but with facial anomalies, including a broad nasal bridge, epicanthus, long deep philtrum and a normal magnetic resonance imaging of the brain. After the examination with MLPA (P036 kit), they were able to detect a terminal deletion in Xp and a duplication of the terminal region of 1q $2.90 \mathrm{Mb}$ in size (Balikova et al., 2007), which was also shared with her mother and grandfather. The carrier patient of 1q duplication had normal stature (75th percentile), despite the deletion of the SHOX gene. In contrast, her mother and her grandfather, did not show features of mental retardation or congenital anomalies due to the 1q terminal duplication and had only short stature as clinical presentation (Balikova et al., 2007). Thus, it is probable that duplication of $\sim 2.20 \mathrm{Mb}$ detected in both cousins did not cause pathogenic effects, as in the family described by Rooms et al. (2006).

This study shows the importance of using molecular techniques such as FISH and MLPA for a more accurate diagnosis, especially in individuals with dysmorphic features and multiple anomalies suggestive of chromosomal changes who have initially shown a normal karyotype. The FISH technique was required to identify the balanced translocation present in the mothers (monozygotic twin sisters) of the patients, indicating the importance of applying molecular cytogenetic techniques in clinical genetics, given the implications for the care of patients and for genetic counseling. In short, our findings contribute to the characterization of a more distal critical region for the cranial dysmorphisms observed in $9 \mathrm{p}$ deletion syndrome and also to the clinical phenotype of patients with monosomy 9p24.3 and trisomy 1q44.

\section{ACKNOWLEDGMENTS}

We thank the Coordenação de Aperfeiçoamento Pessoal de Nível Superior (CAPES) for financial support, Universidade de São Paulo (USP) for logistical support and our patients and their family for their willingness to participate in this study.

\section{REFERENCES}

Alfi O, Donnell GN, Crandall BF, Derencsenyi A, et al. (1973). Deletion of the short arm of chromosome no.9 (46,9p-): a new deletion syndrome. Ann. Genet. 16: 17-22.

Balikova I, Menten B, de Ravel T, Le Caignec C, et al. (2007). Subtelomeric imbalances in phenotypically normal individuals. Hum. Mutat. 28: 958-967.

Barbaro M, Cicognani A, Balsamo A, Lofgren A, et al. (2008). Gene dosage imbalances in patients with 46,XY gonadal 
DSD detected by an in-house-designed synthetic probe set for multiplex ligation-dependent probe amplification analysis. Clin. Genet. 73: 453-464.

Barbaro M, Balsamo A, Anderlid BM, Myhre AG, et al. (2009). Characterization of deletions at 9p affecting the candidate regions for sex reversal and deletion 9p syndrome by MLPA. Eur. J. Hum. Genet. 17: 1439-1447.

Bartsch C, Aslan M, Köhler J, Miny P, et al. (2001). Duplication dup(1)(q32q44) detected by comparative genomic hybridization (CGH): further delineation of trisomies 1q. Fetal Diagn. Ther. 16: 265-273.

Calvari V, Bertini V, De Grandi A, Peverali G, et al. (2000). A new submicroscopic deletion that refines the 9p region for sex reversal. Genomics 65: 203-212.

Chen CP, Su YN, Chern SR, Hsu CY, et al. (2011). Inv dup del(9p): prenatal diagnosis and molecular cytogenetic characterization by fluorescence in situ hybridization and array comparative genomic hybridization. Taiwan. $J$. Obstet. Gynecol. 50: 67-73.

Christ LA, Crowe CA, Micale MA, Conroy JM, et al. (1999). Chromosome breakage hotspots and delineation of the critical region for the 9p-deletion syndrome. Am. J. Hum. Genet. 65: 1387-1395.

Faas BH, de Leeuw N, Mieloo H, Bruinenberg J, et al. (2007). Further refinement of the candidate region for monosomy 9p syndrome. Am. J. Med. Genet. A. 143A: 2353-2356.

Griggs BL, Ladd S, Saul RA, DuPont BR, et al. (2008). Dedicator of cytokinesis 8 is disrupted in two patients with mental retardation and developmental disabilities. Genomics 91: 195-202.

Hauge X, Raca G, Cooper S, May K, et al. (2008). Detailed characterization of, and clinical correlations in, 10 patients with distal deletions of chromosome 9p. Genet. Med. 10: 599-611.

Hoo JJ, Fischer A and Fuhrmann W (1982). Familial tiny 9p/20p translocation: 9p24. The critical segment for monosomy 9p syndrome. Ann. Genet. 25: 249-252.

Huret JL, Leonard C, Forestier B, Rethoré MO, et al. (1988). Eleven new cases of del(9p) and features from 80 cases. $J$. Med. Genet. 25: 741-749.

Kawara H, Yamamoto T, Harada N, Yoshiura K, et al. (2006). Narrowing candidate region for monosomy 9p syndrome to a 4.7-Mb segment at 9p22.2-p23. Am. J. Med. Genet. A. 140: 373-377.

Kulikowski LD, Bellucco FT, Nogueira SI, Christofolini DM, et al. (2008). Pure duplication 1q41-qter: further delineation of trisomy 1q syndromes. Am. J. Med. Genet. A 146A: 2663-2667.

Lenzini E, Ballarati L, Drigo P, Carrozzi M, et al. (2009). 1q44-qter trisomy: clinical report and review of the literature. Genet. Test Mol. Biomarkers 13: 79-86.

Lerer I, Sagi M, Meiner V, Cohen T, et al. (2005). Deletion of the ANKRD15 gene at 9p24.3 causes parent-of-origindependent inheritance of familial cerebral palsy. Hum. Mol. Genet. 14: 3911-3920.

Rooms L, Reyniers E, Wuyts W, Storm K, et al. (2006). Multiplex ligation-dependent probe amplification to detect subtelomeric rearrangements in routine diagnostics. Clin. Genet. 69: 58-64.

Seabright M (1971). A rapid banding technique for human chromosomes. Lancet 2: 971-972.

Shan Z, Zabel B, Trautmann U, Hillig U, et al. (2000). FISH mapping of the sex-reversal region on human chromosome $9 \mathrm{p}$ in two XY females and in primates. Eur. J. Hum. Genet. 8: 167-173.

Shashi V, Berry D, Stamper TH and Pettenati M (1998). A further case of choanal atresia in the deletion (9p) syndrome. Am. J. Med. Genet. 80: 440.

Smith CA, McClive PJ, Western PS, Reed KJ, et al. (1999). Conservation of a sex-determining gene. Nature 402: 601-602.

Swinkels ME, Simons A, Smeets DF, Vissers LE, et al. (2008). Clinical and cytogenetic characterization of 13 Dutch patients with deletion 9p syndrome: Delineation of the critical region for a consensus phenotype. Am. J. Med. Genet. A 146A: $1430-1438$.

Taylor LD, Krizman DB, Jankovic J, Hayani A, et al. (1991). 9p monosomy in a patient with Gilles de la Tourette's syndrome. Neurology 41: 1513-1515.

Verbraak FD, Pogány K, Pilon JW, Mooy CM, et al. (1992). Congenital glaucoma in a child with partial 1q duplication and 9p deletion. Ophthalmic. Paediatr. Genet. 13: 165-170. 